Article

\title{
Coaxial Printing of Silicone Elastomer Composite Fibers for Stretchable and Wearable Piezoresistive Sensors
}

\author{
Zhenhua Tang ${ }^{\circledR}$, Shuhai Jia *, Xuesong Shi, Bo Li and Chenghao Zhou \\ School of Mechanical Engineering, Xi'an Jiaotong University, Xi'an 710049, China; zhtangy@163.com (Z.T.); \\ xuesongshisxs@163.com (X.S.); polee00@163.com (B.L.); francklinson@163.com (C.Z.) \\ * Correspondence: shjia@mail.xjtu.edu.cn
}

Received: 15 March 2019; Accepted: 9 April 2019; Published: 11 April 2019

\begin{abstract}
Despite the tremendous efforts dedicated to developing various wearable piezoresistive sensors with sufficient stretchability and high sensitivity, challenges remain pertaining to fabrication scalability, cost, and efficiency. In this study, a facile, scalable, and low-cost coaxial printing strategy is employed to fabricate stretchable and flexible fibers with a core-sheath structure for wearable strain sensors. The highly viscous silica-modified silicone elastomer solution is used to print the insulating sheath layer, and the silicone elastomer solutions containing multi-walled carbon nanotubes (CNTs) are used as the core inks to print the conductive inner layer. With the addition of silica powders as viscosifiers, silica-filled silicone ink (sheath ink) converts to printable ink. The dimensions of the printed coaxial fibers can be flexibly controlled via adjusting the extrusion pressure of the inks. In addition, the electro-mechanical responses of the fiber-shaped strain sensors are investigated. The printed stretchable and wearable fiber-like CNT-based strain sensor exhibits outstanding sensitivities with gauge factors (GFs) of 1.4 to $2.5 \times 10^{6}$, a large stretchability of $150 \%$, and excellent waterproof performance. Furthermore, the sensor can detect a strain of $0.1 \%$ and showed stable responses for over 15,000 cycles (high durability). The printed fiber-shaped sensor demonstrated capabilities of detecting and differentiating human joint movements and monitoring balloon inflation. These results obtained demonstrate that the one-step printed fiber-like strain sensors have potential applications in wearable devices, soft robotics, and electronic skins.
\end{abstract}

Keywords: coaxial printing; carbon nanotube; silicone elastomer; strain sensor; human motion monitoring

\section{Introduction}

Soft and flexible piezoresistive sensors, as a key component of soft electronic devices, have recently become prevalent in various research fields, such as soft robotics, wearable electronics, healthy monitoring, and human-machine interfaces [1]. In particular, stretchable fiber-based sensors, which are expected to be flexible, wearable, and light-weight, are promising as a platform for wearable electronic devices [2]. Furthermore, a fibrous or wire-shaped device can be easily integrated into stretchable fabrics to fulfill a more practical demand of wearable electronics in our daily life [3]. Therefore, tremendous efforts have been made to develop fiber-like piezoresistive sensors [4-7].

Recently, conductive materials such as carbon nanotubes (CNTs) [8], graphene [9], liquid alloy [10], and metal nanowire solutions [11] infiltrated into or coated on stretchable elastomer fibers have been widely used to fabricate fiber-shaped sensors. For example, Cao et al. [11] demonstrated a silver nanowire/polyurethane composite fiber sensor with high sensitivity, but the fabricate method is complex. Zhang et al. [12] fabricated a highly stretchable conductive fiber by dip-coating a layer 
of liquid metal on silicone elastomer filaments, but the separation between the surface layer and the filament as the fiber bends, stretches, and shrinks is inevitable. To obtain a high-performance wire-like strain sensor, Boland et al. reported a simple method to fabricate graphene-infused elastic bands to produce highly stretchable and sensitive strain sensors [9]. On the other hand, soft and elastic tubes encapsulating conductive fillers have also been used to fabricate fiber-like piezoresistive sensors. For instance, conductive stretchable fibers were fabricated by injecting liquid alloys into elastic polymer tubes [10]. Although large stretchability was achieved, the injection method and the liquid leakage may limit the practical application of this type of fiber-like sensor. Luo et al. [13] filled expanded graphite into an elastic rubber tube to fabricate a high-performance stretchable tubular conductor, but this fabrication strategy is uncontrollable. Zhou et al. [14] combined the wet-spinning approach with a post-treatment process to prepare thermoplastic elastomer-wrapped CNT fiber-like strain sensors, which exhibited high sensitivity, high stretchability, and high linearity, but the fabrication process was complex and time-consuming. In our previous study [5,15], CNT-based coaxial fibers were fabricated via a one-step wet-spinning assembly approach. The sheath layer and core layer of the fibers were pure silicone elastomer and CNT-filled silicone elastic composite, respectively. Due to the elastic nature of silicone elastomer, the stretchability of the coaxial fiber was above $500 \%$. Moreover, the insulating property of the sheath layer of the coaxial fiber avoided the risk of short-circuiting. Various conductive materials and fabrication methods have been employed to fabricate fiber-based piezoresistive sensors. However, most conducting fibers in previous studies were fabricated by physically deposited fillers or solution-coated conducting materials [7-9,12]. The common methods such as dip-coating, spray-coating, and a layer-by-layer assembly method have some limitations, such as complex processes, high costs, and manual interventions. Moreover, the conductive surfaces of the aforementioned fibers being exposed and the risk of short-circuiting when used as strain sensors needed to be considered $[4,6-9,12]$. The effect of sweat and water in the vicinity on the sensors are also challenges for practical applications. Therefore, the development of wearable and waterproof fiber-like sensors via a simple, efficient, and scalable fabrication process still needs to be addressed. Direct ink writing (DIW) is one of the 3D printing techniques that has drawn much attention, due to its simple printing mechanisms, low cost, and large-scale production. DIW has been applied to print sensors, actuators, energy storage devices, and so on [16-18]. For example, Wang et al. [19] fabricated $\mathrm{CNT} /$ polydimethylsiloxane(PDMS) strain sensors by layer-by-layer printing CNT dispersion on a PDMS substrate. Kim et al. [20] employed the DIW technique to fabricate eutectic gallium-indium (EGaIn)-based soft sensors. More recently, Cheng et al. [21] fabricated hybrid solid-state electrolytes by using the DIW technique without any additional processing steps. These explorations show that DIW has huge potential in fabricating advanced devices with low-cost, high-efficiency, and mass production properties.

Here, we employed DIW printing technology to print coaxial CNT-based polymeric composites for superelastic fiber-shaped piezoresistive sensors. The core layer ink was composed of CNTs and silicone elastomer solution, and the sheath layer ink was a viscoelastic silica-filled silicone elastomer mixture. Silica nanoparticles were added into the silicone elastomer solutions to modify their rheological properties and reinforce the mechanical properties. A coaxial nozzle was used in this method, of which the out nozzle and inner nozzle were used to extrude the silica-filled silicone elastomer and $\mathrm{CNT} /$ silicone inks, respectively. Appropriate process parameters were selected to ensure a successful printing process. The printed coaxial fibers exhibited excellent mechanical and electrical properties, which could be used as stretchable and wearable piezorisitive sensors. The printed fiber-like sensor exhibited an ultrahigh sensitivity (gauge factor (GF) of $2.5 \times 10^{6}$ at a strain of 90-150\%), a large stretchability $(150 \%)$, and excellent durability and repeatability (over 15,000 cycles). Furthermore, we demonstrated the waterproof property and the detection of various human motions with the printed sensor. The combination of one-dimensional (1D) coaxial fiber design and the easy, low-cost, and scalable DIW printing technology can offer a promising solution for wearable and high-performance electronic devices. 


\section{Materials and Methods}

\subsection{Materials and Characterizations}

Unless otherwise specified, all materials were used as received. Multi-walled carbon nanotubes (CNTs, average diameter: $12 \mathrm{~nm}$, length: 10-30 $\mu \mathrm{m}$, and purity: $>98 \%$ ) were purchased from Chengdu Organic Chemicals Co., Ltd. of the Chinese Academy of Science, Chengdu, China. Silica nanoparticles (Si NPs) with 7-40 nm particle size and $300 \mathrm{~m}^{2} / \mathrm{g}$ specific surface area were supplied by Shanghai Aladdin Biochemical Technology Co., Ltd. Commercially available silicone elastomer Ecoflex 0030 was purchased from Smooth-On (Macungie, PA, USA). The surface morphology of CNTs was characterized by a field-emission scanning electron microscope (SEM) (Zeiss GenimiSEM 500, Oberkochen, Germany). The quality of CNTs was analyzed by Raman spectroscopy HR800 (Jobin Yvon Horiba, Paris, France) with a laser excitation wavelength of $633 \mathrm{~nm}$. The sizes of the fibers were obtained using an optical microscope (GP-300C, Kunshan Gaopin Precision Instrument Co., Ltd., Kunshan, China).

\subsection{Preparation of the Printable Inks}

Homogeneous printable inks were prepared as follows: The sheath silicone elastomeric ink was synthesized by mixing 1 Ecoflex 0030 Part A to 1 Part B with a certain amount of Si NP, which was added as a rheological modifier [22]. To achieve appropriate rheological properties for a 3D printing ink, the Si NP content of the sheath ink was optimized to be $5.5 \mathrm{wt} \%$. The core inks were prepared as follows: Ecoflex 0030 Part A and B were mixed at a 1:1 ratio, followed by the addition of a proper amount of CNT powders. The inks were mixed using a planetary centrifugal mixer (HM800, Shenzhen Hasai Technology Co., Ltd., Shenzhen, China) at $2000 \mathrm{rpm}$ for $5 \mathrm{~min}$.

\subsection{Rheological Measurements}

The rheological properties of the inks were characterized using a stress-controlled rheometer (MCR302, Anton Paar, Graz, Austria) with a 25-mm diameter parallel plate at room temperature. The viscosity of the inks was measured through a shear-rate sweep from 0.1 to $100 \mathrm{~s}^{-1}$. Dynamic stress sweeps were performed at a fixed frequency of $1 \mathrm{~Hz}$ to get storage modulus $\left(\mathrm{G}^{\prime}\right)$ and loss modulus $\left(\mathrm{G}^{\prime \prime}\right)$ as a function of the shear stress sweep from 0.1 to $1000 \mathrm{~Pa}$.

\subsection{Coaxial Printing}

Coaxial printing was carried out using a homemade 3D printing system, which consisted of a computer-controlled 3-axis movement platform. All printing paths were determined using G-code commands, which were generated by commercial software (CuraEngine, Geldermalsen, The Netherlands) from designed 3D models (SolidWorks, MA, USA). Two $20 \mathrm{~mL}$ pneumatic syringes were used to store the core and sheath inks individually. An air cylinder connected to an air compressor and two pressure regulators were used to provide the appropriate pressure to extrude the ink through the coaxial nozzle. The inks were extruded through a coaxial nozzle at a distance of $1.5 \mathrm{~mm}$ from the substrate. The coaxial printing nozzle was fabricated by inserting a $19 \mathrm{G}$ stainless steel needle (diameter: $0.67 \mathrm{~mm}$ ) into a $13 \mathrm{G}$ stainless steel needle (diameter: $1.9 \mathrm{~mm}$ ). The usual printing speed was about $2 \mathrm{~mm} \mathrm{~s}^{-1}$, and the printing pressure of the sheath ink was about $0.64 \mathrm{MPa}$, which were the optimized parameters. The flow rate of the core ink was controlled by an air pressure governing valve that could flexibly adjust the pressure (range from 10 to $90 \mathrm{kPa}$ ) of the air to drive the inks. After printing, the final obtained fibers were fully dried in an oven at $40{ }^{\circ} \mathrm{C}$ for $1 \mathrm{~h}$. Unless otherwise specified, the samples for all experiments in this research were printed at $70 \mathrm{kPa}$ and $0.64 \mathrm{MPa}$ for core ink and sheath ink, respectively. 


\subsection{Elecreomechanical Response Measurement}

Copper wires were connected to the two ends of the printed coaxial fiber as external electrodes with the help of conductive silver paste and silicone adhesive (Sil-Poxy, Smooth-On). The gauge length between the copper wires was $20 \mathrm{~mm}$. The silicone adhesive was used to cover the silver electrodes to avoid mechanical failure between the soft fiber and rigid electrodes. A computer-controlled homemade stretching stage was used to apply the desired strains. Piezoresistive responses of the printed sensors were measured by recording the current at a constant voltage of $1 \mathrm{~V}$. The electrical responses of the sensors were acquired with a data acquisition module (NI USB-6341, National Instruments, Austin, TX, USA) and transmitted to a computer. All experiments in this study were conducted at room temperature (about $24^{\circ} \mathrm{C}$ ).

\section{Results}

Figure 1a shows the SEM image of the pristine CNT powders, which had an average diameter of $12 \mathrm{~nm}$. Figure $1 \mathrm{~b}$ shows the Raman spectrum for the raw CNTs. The D peak at $1320 \mathrm{~cm}^{-1}, \mathrm{G}$ peak at $1580 \mathrm{~cm}^{-1}$, and the 2D peak at $2640 \mathrm{~cm}^{-1}$ were observed. The $\mathrm{D}$ peak is attributed to the disorder of the carbonaceous structures. The $\mathrm{G}$ peak is associated with the $\mathrm{sp}^{2}$ vibration of a perfect graphite crystal [23]. In general, the less disordered the graphite-based systems are, the weaker the intensity of the D peak (relative to the intensity of the G peak) is expected to be. As shown in Figure 1b, the calculated intensity ratio of the $D$ peak to the $G$ peak $\left(I_{D} / I_{G}\right)$ turned out to be about 1.5 , indicating a high level of impurity or defect density in the purchased CNTs. To prepare inks for printing, the rheological properties should be taken into account.Figure $1 \mathrm{c}$ shows the curves of viscosity as a function of shear rate for various inks. The apparent viscosity value of pure silicone solutions is less than $10 \mathrm{~Pa} \cdot \mathrm{s}$, which is much lower than the printable inks we used, indicating inferior printability. Unlike the pure silicone inks, the addition of CNTs or Si NPs makes the silicone-based composite inks exhibit a remarkable shear-thinning behavior, which is critical for controllable extrusion during printing. Figure $1 \mathrm{~d}$ shows the storage modulus $\left(G^{\prime}\right)$ and loss modulus $\left(G^{\prime \prime}\right)$ as a function of shear stress for various inks. Storage modulus describes the solidification behavior of the ink in low shear stress conditions, while the loss modulus reflects the liquid-like response. A high storage modulus at low shear stress helped the inks retain their filamentary form after printing. For sheath ink, the content of Si NPs for sheath inks was optimized to be $5.5 \mathrm{wt} \%$, considering the high shape retention at various printing conditions. As shown in Figure 1d, the storage modulus $\left(\mathrm{G}^{\prime}\right)$ of pure silicone solution is much lower than its loss modulus $\left(G^{\prime \prime}\right)$, indicating a liquid-like behavior. The storage modulus $\left(G^{\prime}\right)$ of silica-filled silicone inks plateaus above $10^{4} \mathrm{~Pa}$ which is much higher than the relevant loss modulus range. These results indicate that silica-filled silicone inks were transformed into a solid-like fluid, due to the formation of a strong silica network at this filler loading, which facilitated the shape retention of the printed patterns. Moreover, the core ink shows a high $\mathrm{G}^{\prime}$ over $10^{3} \mathrm{~Pa}$, which is higher than $\mathrm{G}^{\prime \prime}$ in the low shear stress region. This result indicated that the $\mathrm{CNT} /$ silicone ink was also transformed into a solid-like fluid, due to the interaction between CNTs and polymer chains. It should be noticed that the $\mathrm{G}^{\prime}$ and $\mathrm{G}^{\prime \prime}$ of the sheath ink are much higher than those of the core ink. The higher $\mathrm{G}^{\prime}$ indicated a stiffer nature of the silica-filled silicone ink, which is desirable for printing self-supported structures without deformation. In fact, owing to the coaxial structural feature of the fiber composite, the higher $\mathrm{G}^{\prime}$ of the sheath ink was able to lower the requirements for rheological properties of the core ink. That is to say, the rheological properties of the core inks don't need to meet the printing requirements (shear-thinning and larger storage modulus) in this study. 
a



C

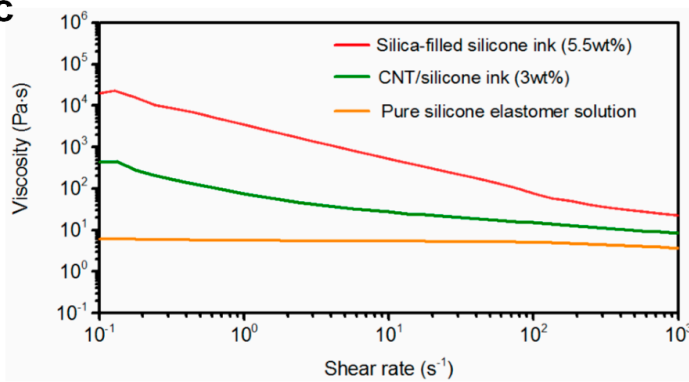

b



d

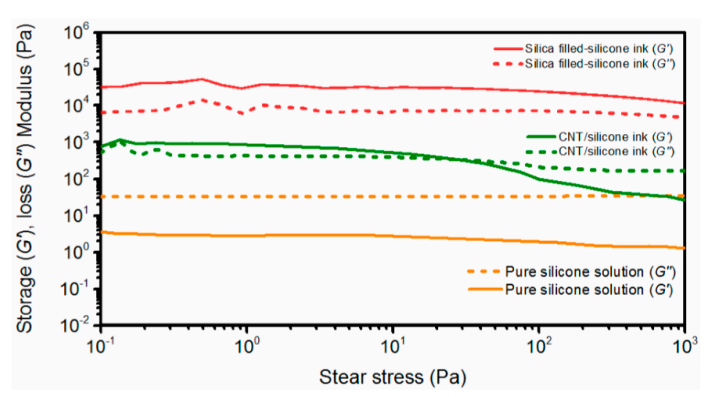

Figure 1. (a) SEM image of carbon nanotubes (CNTs). (b) Raman spectra for raw CNTs. (c) Apparent viscosity as a function of shear rate for various inks. (d) Storage modulus $\left(G^{\prime}\right)$ and loss modulus $\left(G^{\prime \prime}\right)$ as a function of shear stress for various inks.

Figure 2a briefly shows the preparation process of the silicone-based inks. The silica-filled silicone ink for the sheath layer was formulated by simply mixing Si NPs and silicone solution in a certain weight proportion to create a uniformly dispersed and high-viscosity composite ink. Similarly, the core layer ink was fabricated by mixing CNTs and silicone solution in a certain weight content. The prepared silica-filled silicone ink and CNT/silicone ink were mixed using a planetary centrifugal mixer at $2000 \mathrm{rpm}$ for $5 \mathrm{~min}$. Then the prepared inks were housed in separate syringes ( $20 \mathrm{~mL}$ volume) for coaxial printing. As shown in Figure 2b, the loaded syringes attached with the coaxial nozzle were then mounted onto the specially designed 3D DIW printer. The coaxial printing process is illustrated in Figure 2c. Figure $2 d$ shows the optical images of the loaded syringes with the prepared inks. Figure $2 \mathrm{e}$ shows the optical image of the extruding process of the inks through the coaxial nozzle. From this figure, it can be seen that the extruded filament exhibited a clear core-sheath structure and could maintain its shape without breakdown, indicating the printability of the inks. The flow rates of core layer ink and sheath layer ink were separately controlled by air pressure governing valves that could flexibly adjust the injection pressure of air to drive the inks. The movement speed of the nozzle and the printing pressure of the sheath ink were about $2 \mathrm{~mm} \mathrm{~s}^{-1}$ and $0.64 \mathrm{MPa}$, respectively, which were the optimized parameters. The pressure of the core layer ink was adjusted from 10 to $90 \mathrm{kPa}$. Figure $2 \mathrm{f}$ shows the digital optical image of the printing process of a coaxial fiber. Finally, the printed samples were cured at $40^{\circ} \mathrm{C}$ for $1 \mathrm{~h}$ and removed from the substrate after cooling down to room temperature. Figure $2 \mathrm{~g}$ shows that the printed fibers can be stretched to above $100 \%$ of tensile strain, demonstrating their high flexibility and stretchability.

Figure 3a shows the optical microscopy images of the transverse cross-sectional areas of the printed fibers. From this figure, it can be seen that the printed coaxial fibers consisted of conductive $\mathrm{CNT} /$ silicone elastomer composites (black areas), which were encapsulated by elastomeric insulating layers made up of fumed silica-filled silicone elastomer. The cross-section areas were measured form the optical microscopy images with respect to different extrusion pressures. Figure $3 \mathrm{~b}$ shows the diameter variation of the printed samples under different extrusion pressures of core inks. From this figure, it can be noted that the core diameter of the coaxial fibers increased with the corresponding extrusion pressure. The diameters of the coaxial fibers, however, show only a slight variation, as 
depicted in Figure $3 \mathrm{~b}$. The total diameter of the fiber slightly increased when increasing the core ink injection pressure to approximately $50 \mathrm{kPa}$, and then the fiber diameter decreased slightly with the increasing pressure. This may be attributed to the thickness of the sheath layer decreasing as the diameter of the core layer increased. The higher the extruding pressure of core ink, the larger the core diameter, but increasing the core ink injection pressure too much would result in the thickness of the sheath layer decreasing quickly. Therefore, the total diameter of the printed fibers exhibited a reducing trend under a certain high extrusion pressure.
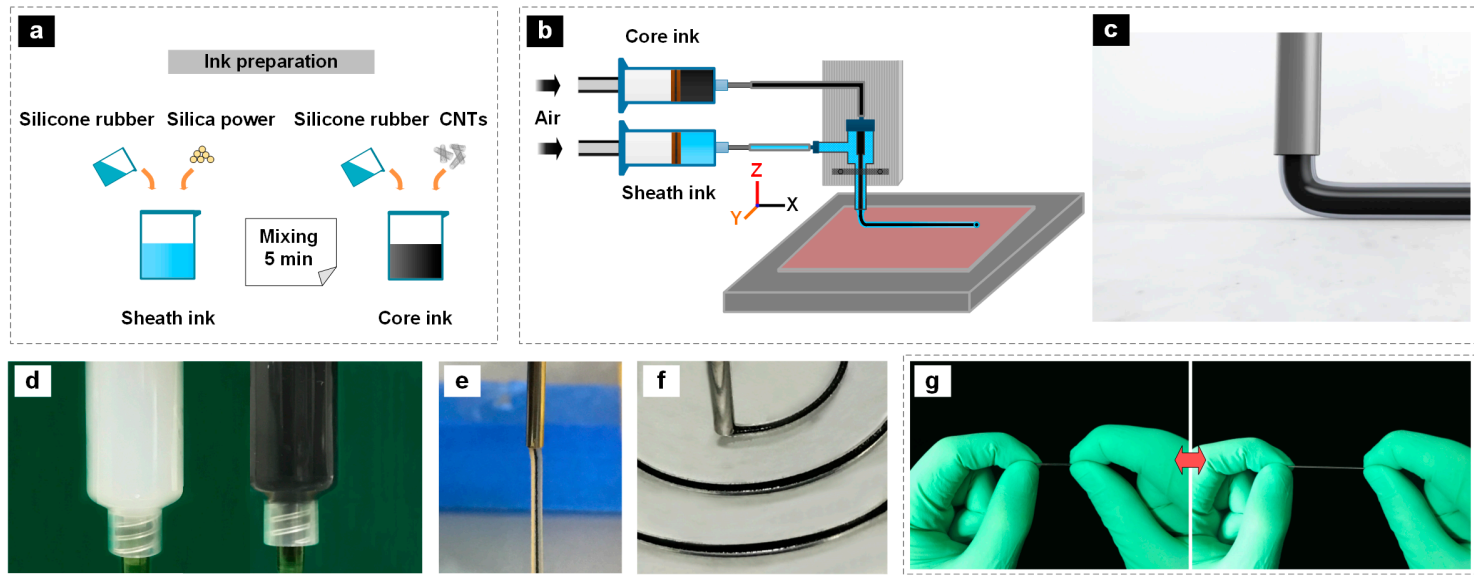

Figure 2. (a) Schematic showing the preparation of the printable inks. (b) Schematic illustration of the coaxial printing system. (c) Schematic illustration of the coaxial printing process and the coaxial structure of the printed fiber. (d) Digital optical images of silica-filled silicone ink (left) and CNT/silicone ink (right). (e) Digital optical image of ink-extruding. (f) Photograph showing the coaxial printing process. (g) Digital optical images of the printed fiber under original (left) and stretched (right) state, showing the excellent stretchability of the fiber.

a
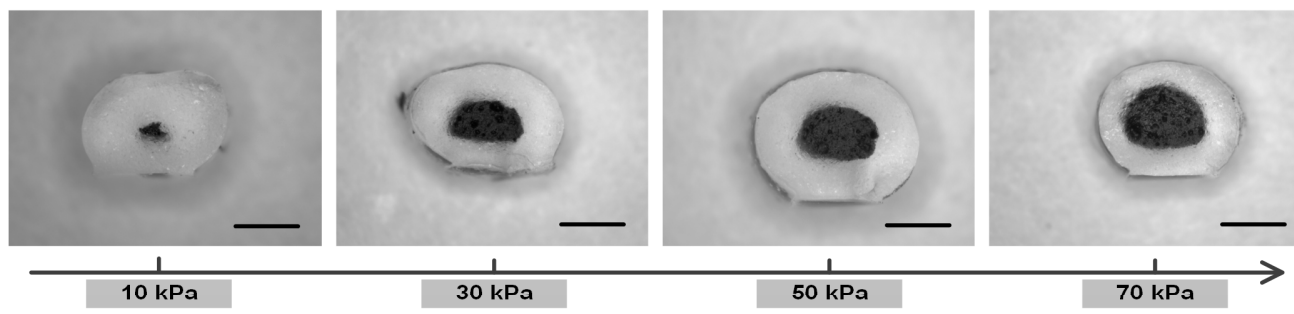

b

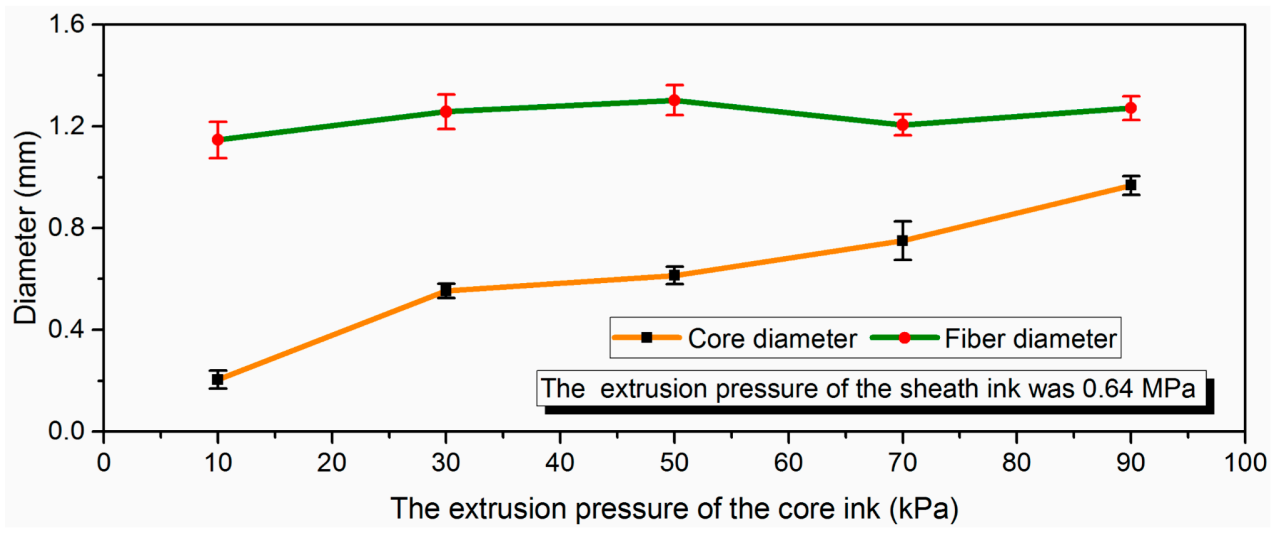

Figure 3. (a) Optical microscopy images of the transverse cross-sectional areas of the printed fibers with respect to different injection pressures. The extrusion pressure of the sheath ink remained constant (0.64 MPa). Five samples were measured for each fiber type. All scale bars represent $0.5 \mathrm{~mm}$. (b) The whole size of the fiber and the core diameter of the fiber as a function of the inner layer injection pressure. 
Figure 4 shows the current-voltage $(I-V)$ curves of the printed fiber-like sensor under various static strains, ranging from 0 to $40 \%$. From this figure, it can be noted that the $I-V$ curves all appeared to have a linear tendency, indicating the ohmic behavior and constant conductivity of the sensor under static loading. Therefore, the resistance of the device is independent of the applied voltage. The piezoresistive behavior of the sensor was investigated by monitoring the relative changes in electrical resistance for the applied strain. The resistance of the sensor increased with the applied strain, and the electric connection was lost under a strain above $150 \%$. It should be noted that the fracture strain of the coaxial fiber was about $400 \%$. Figure $4 \mathrm{~b}$ shows the relative changes in electrical resistance $\left(\Delta R / R_{0}\right)$ of the sensor under various strains. The gauge factor (GF) is a characteristic parameter representing the sensitivity of the sensor and can be calculated from $\left(\Delta R / R_{0}\right) / \varepsilon$, where $\Delta R, R_{0}$, and $\varepsilon$ denote the change in resistance, initial resistance, and applied strain, respectively. The calculated GFs of the sensor were 1.4 and $2.5 \times 10^{6}$ for strains ranging from 0 to $25 \%$ and 90 to $150 \%$, respectively. Figure $4 \mathrm{c}$ shows the normalized resistance changes of the sensor under various cyclic strains of $20 \%, 40 \%, 60 \%$, and $80 \%$. From this figure, it can be noted that the sensor exhibits a uniform and repeated response according to the applied strain. A shoulder peak pattern was observed during strain releasing. The shoulder peak originates from the competition between the destruction and reconstruction of the conductive networks during the releasing process. A similar behavior has been previously reported [24,25]. Here, the shoulder peaks for larger strain of $60 \%$ and $80 \%$ were negligible. In addition, as shown in Figure $4 \mathrm{~d}$, the sensor could successfully differentiate strain from $0.1 \%$ to $0.5 \%$, indicating the capability of detecting subtle strains. The dynamic response of the sensor under a square wave loading signal is shown in Figure 4e. From this figure, it can be seen that the sensor exhibited a consistent change in resistance, indicating excellent repeatability. During stretching, the response of the sensor showed overshooting in response to acceleration. A similar phenomenon has been previously reported for CNT-based polymer composites $[5,26]$. This behavior may be attributed to the viscoelasticity of the silicone elastomer. Figure $4 \mathrm{f}$ shows the response time of the sensor. The response time is estimated to be around $300 \mathrm{~ms}$. If the relay of the measurement system were considered, the actual response time for the sensor itself would be even shorter than this value. For the frequency response test, we applied a strain of $50 \%$ at a varying strain rate of 0.5 to $3.6 \mathrm{~Hz}$. Figure $4 \mathrm{~g}$ shows the relative change of the resistance for the sensor at various frequencies. From this figure, it can be noted that the sensor retained its performance until a strain rate of $3.6 \mathrm{~Hz}$, showing a stable variation in resistance. The relative resistance change slightly increases with an increase of the frequency of the applied strain. This may be attribute to that the higher strain rate causes greater stress in the materials, which leads to an increase in the amplitude of relative resistance change at high frequencies [5]. Moreover, the dynamic durability of the sensor was investigated by monitoring the response of the sensor under cyclic stretching at a strain of $50 \%$ at $1 \mathrm{~Hz}$. As shown in Figure $4 \mathrm{~h}$, the sensor maintained the sensing performance for 15,000 cycles, indicating that the sensor had a long working life and excellent repeatability.

The stability of the sensor in a wet environment is necessary for practical application. The electrical response of the sensor to water was monitored for one hour by soaking the sensor in water (Figure 5a). Figure $5 \mathrm{~b}$ shows the relative changes in resistance of the sensor as a function of the soaking time. From this figure, it can be noted that there were no significant changes in the electrical resistance after soaking in water for $60 \mathrm{~min}$. In addition, the sensor was attached onto the back of the index finger by bonding the two ends with a stretchable tape (Figure 5c), and the dynamic response of the sensor in water at room temperature was recorded. The electrical resistance was recorded by the source meter (Model 2450, Keithley, Cleveland, OH, USA). Owing to the flexibility of the sensor, conformal attachment to uneven skin can be achieved. Figure $5 \mathrm{~d}$ shows the relative changes in resistance of the sensor during the bending motions of the finger in air and water at room temperature. From this figure, it is clearly seen that the dynamic responses of the sensor in air and water were similar, indicating that the sensor exhibited excellent waterproof properties. This can be attributed to the conductive core being fully sealed with hydrophobic silicone rubber, which prevented water molecules from being absorbed into the sensor surface. Therefore, the sheath layer effectively reduced the effect of the water on the sensors. 

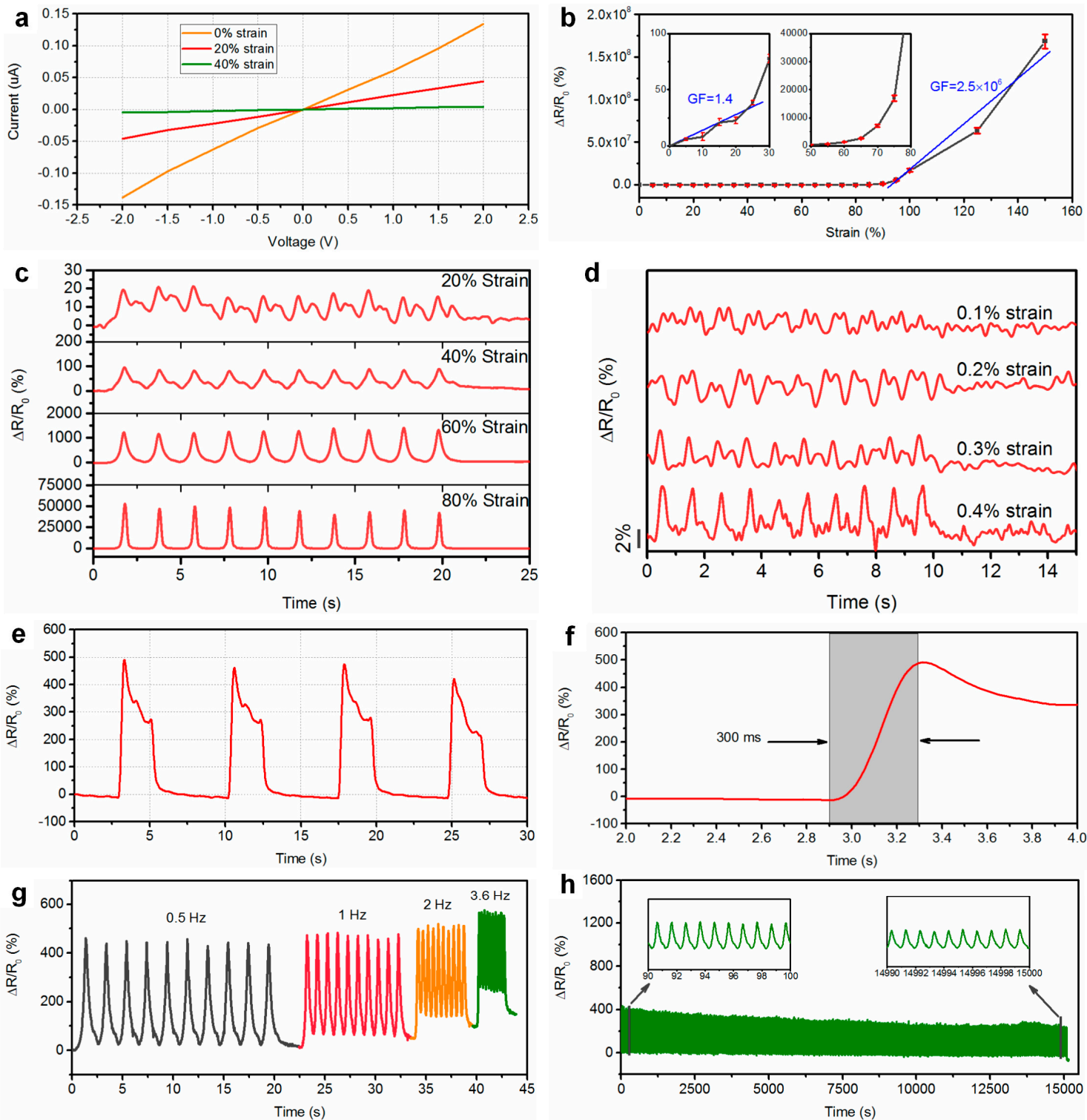

Figure 4. (a) Current-voltage $(I-V)$ curves of the printed sensor with applied various strains. (b) Normalized resistance changes of the sensor under tensile strain. The insets show the normalized resistance changes of the sensor at low strain ranges. (c) Dynamic responses of the sensor to repeated strains of $20 \%, 40 \%, 60 \%$, and $80 \%$. (d) Normalized resistance changes of the sensor under strains of 0.1 to $0.4 \%$. (e) Normalized resistance changes of the sensor upon applying a quasi-transient step strain from $\varepsilon=0 \%$ to $\varepsilon=50 \%$. (f) Magnified sensor responses extracted from (e) to show the response time. (g) Relative resistance changes of the sensor vs. a tensile strain of $50 \%$ at frequencies of $0.5,1,2$, and $3.6 \mathrm{~Hz}$. (h) Normalized resistance changes of the sensor under repeated stretching/releasing cycles with $50 \%$ strain at a frequency of $1 \mathrm{~Hz}$ for 15,000 cycles, demonstrating the durability of the printed sensor. The insets show the normalized resistance changes of the sensor from 90 to $100 \mathrm{~s}$ and 1490 to $1500 \mathrm{~s}$. 

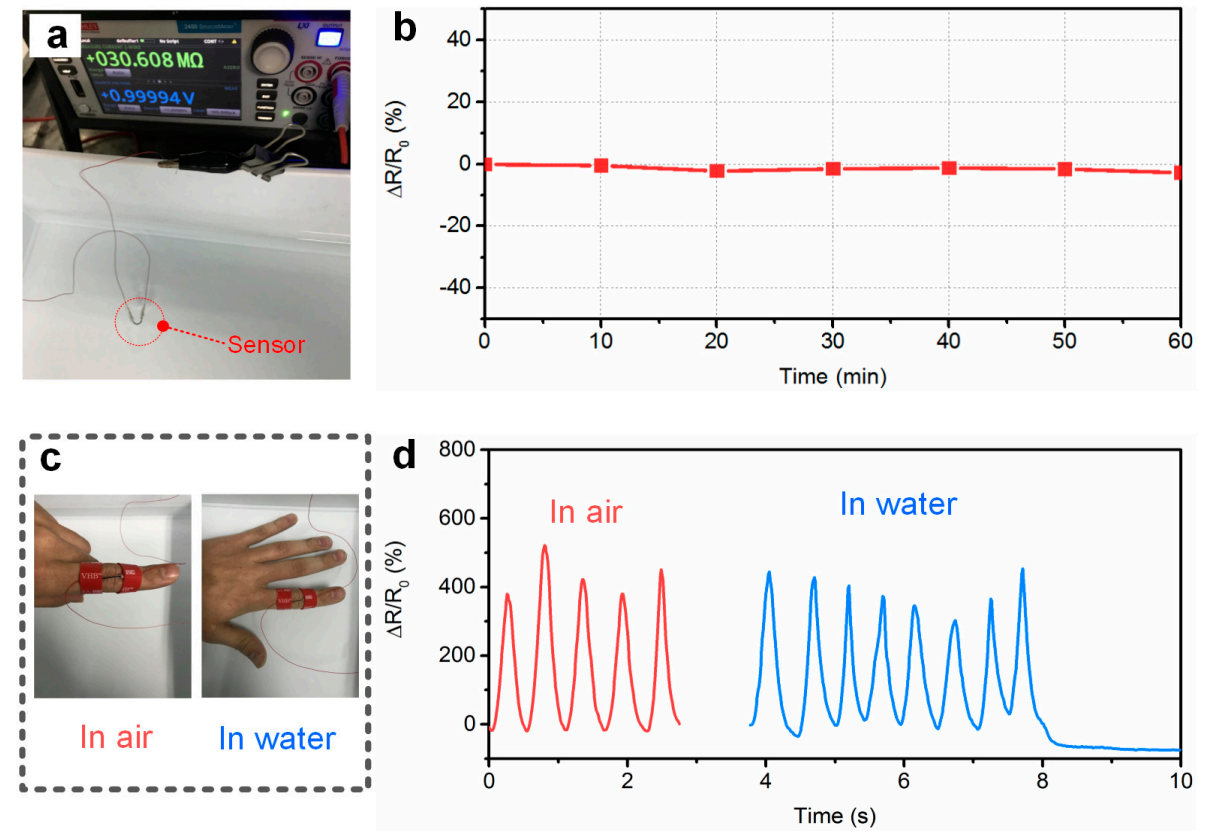

Figure 5. (a) The fiber sensor system while soaking in water. (b) Relative changes in electrical resistance of the sensor as a function of the soaking time. (c) Photographs of the sensor directly attached to the index finger. (d) A comparison of the dynamic responses of the printed sensors during finger bending movements in air and water at room temperature.

Furthermore, to demonstrate the potential of the printed fibers as wearable sensors, the fiber-shaped sensor was woven into the index finger of a fabric glove, using a sewing method. Figure 6a shows the photographs of a fiber sensor woven into a glove. Figure $6 \mathrm{~b}$ shows the relative changes in resistance of the sensor under cyclic bending motions (various bending angles). From this figure, it can be seen that the sensor responded to the motion of the finger quickly and accurately. Moreover, the sensor reliably detected resistance changes, depending on the degree of bending of the finger, and the sensor distinguishably responded to different finger motions. Figure $6 \mathrm{c}$ shows dynamic resistance changes of the sensor under consecutive step-and-hold tests. From this figure, it can be seen that the resistance variation increased in a stepwise manner in real time as the bending angle of the finger increased step by step. When the finger recovered to its original state gradually, the resistance decreased according to the bending angles. The above results fully indicate that the as-printed sensor with high sensitivity and excellent stability presents potential applications for monitoring and analyzing human motions.

Thanks to the excellent flexibility and ultra-light weight of the fiber, the sensors could be attached conformally to various uneven surfaces. As a demonstration, the sensor was directly attached to the surface of a flat balloon (Figure 6d). The balloon was blown to expand its surface, while the electrical resistance was recording in real-time. Figure 6e shows the resistance changes of the sensor, depending on the gas volume in the balloon. From this figure, it can be seen that the response curve of the sensor in a stair-like pattern corresponds to the process of the blowing. When the expanding balloon deflated, the electrical resistance reduced. Based on the above results and the wide sensing range and remarkable sensitivity of the sensors, we believe that the printed fiber-like sensor demonstrates its high potential for application as a wearable strain sensor. 



Figure 6. (a) Optical images of a sensor woven into a glove at different bending angles. (b) Relative changes in resistance for bending/unbending motions of an index finger with various bending angles. (c) Electrical resistance responses of the sensor under consecutive step-and-hold tests. (d) Photographs of a strain sensor attached to a balloon at various inflating states. All scale bars represent $20 \mathrm{~mm}$.

(e) Monitoring of tension changes on the balloon surface during inflation.

\section{Conclusions}

In summary, we demonstrated a simple and efficient strategy to construct a stretchable and flexible fiber-shaped strain sensor with both high sensitivity and large sensing range, through a coaxial printing technique. A series of novel printable silicone elastomer-based inks with shear-thinning behavior was developed. Through the addition of fumed silica powders or CNTs as viscosifiers, silicone elastomer-based inks became adaptable for DIW printing, and coaxial fibers were successfully printed with high-throughput production capability. The printed coaxial fibers exhibited high stretchability and excellent flexibility, which were used as wearable strain sensors. It was demonstrated that the fiber-like strain sensors showed high sensitivity (maximum gauge factor of $2.5 \times 10^{6}$ at 90 to $150 \%$ strain), large sensing area (0-150\% strain), outstanding durability (15,000 cycles), and excellent waterproof performance. Furthermore, the printed coaxial fiber-based sensors were proven to be capable of detecting and differentiating human joint motions and other curved surface deformations. The one-step coaxial printing and synthesis of the inks in this work could provide inspiration for the development of conformal and readily fabricated lightweight wearable electronics.

Author Contributions: Conceptualization, Z.T. and S.J.; Methodology, Z.T., X.S. and C.Z.; Validation, X.S., S.J., and B.L.; Investigation, Z.T. and C.Z.; Writing-Original Draft Preparation, Z.T.; Writing-Review \& Editing, Z.T.; Project Administration, S.J.; Funding acquisition, S.J.

Funding: This work is supported by the National Natural Science Foundation of China (NSFC) (No. 51575437), the Key Science and Technology Program of Shaanxi Province of China (No. 2014K07-02), and the XJTU University Funding (No. PY3A048).

Acknowledgments: This work is supported by the National Natural Science Foundation of China (NSFC) (No. 51575437), the Key Science and Technology Program of Shaanxi Province of China (No. 2014K07-02), and the XJTU University Funding (No. PY3A048). The authors thank Zijun Ren at Instrument Analysis Center of Xi'an Jiaotong University for his assistance with SEM characterization. 
Conflicts of Interest: The authors declare no conflict of interest.

\section{References}

1. Amjadi, M.; Kyung, K.U.; Park, I.; Sitti, M. Stretchable, skin-mountable, and wearable strain sensors and their potential applications: A review. Adv. Funct. Mater. 2016, 26, 1678-1698. [CrossRef]

2. Zeng, W.; Shu, L.; Li, Q.; Chen, S.; Wang, F.; Tao, X.M. Fiber-based wearable electronics: A review of materials, fabrication, devices, and applications. Adv. Mater. 2014, 26, 5310-5336. [CrossRef]

3. Varma, S.J.; Kumar, K.S.; Seal, S.; Rajaraman, S.; Thomas, J. Fiber-type solar cells, nanogenerators, batteries, and supercapacitors for wearable applications. Adv. Sci. 2018, 5, 1800340. [CrossRef] [PubMed]

4. Liu, Z.; Qi, D.; Hu, G.; Wang, H.; Jiang, Y.; Chen, G.; Luo, Y.; Loh, X.J.; Liedberg, B.; Chen, X. Surface strain redistribution on structured microfbers to enhance sensitivity of fiber-shaped stretchable strain sensors. Adv. Mater. 2017, 1704229.

5. Tang, Z.; Jia, S.; Wang, F.; Bian, C.; Chen, Y.; Wang, Y.; Li, B. Highly stretchable core-sheath fibers via wet-spinning for wearable strain sensors. ACS Appl. Mater. Interfaces 2018, 10, 6624-6635. [CrossRef]

6. Jeong, S.M.; Kang, Y.; Lim, T.; Ju, S. Hydrophobic microfber strain sensor operating stably in sweat and water environment. Adv. Mater. Interfaces 2018, 5, 1801376. [CrossRef]

7. Yu, S.; Wang, X.; Xiang, H.; Zhu, L.; Tebyetekerwa, M.; Zhu, M. Superior piezoresistive strain sensing behaviors of carbon nanotubes in one-dimensional polymer fiber structure. Carbon 2018, 140, 1-9. [CrossRef]

8. Wang, Z.; Huang, Y.; Sun, J.; Huang, Y.; Hu, H.; Jiang, R.; Gai, W.; Li, G.; Zhi, C. Polyurethane/cotton/carbon nanotubes core-spun yarn as high reliability stretchable strain sensor for human motion detection. ACS Appl. Mater. Interfaces 2016, 8, 24837-24843. [CrossRef]

9. Boland, C.S.; Khan, U.; Backes, C.; O’Neill, A.; McCauley, J.; Duane, S.; Shanker, R.; Liu, Y.; Jurewicz, I.; Dalton, A.B.; et al. Sensitive, High-strain, high-rate bodily motion sensors based on graphenerubber composites. ACS Nano 2014, 8, 8819-8830. [CrossRef]

10. Yu, L.; Yeo, J.C.; Soon, R.H.; Yeo, T.; Lee, H.H.; Lim, C.T. Highly stretchable, weavable, and washable piezoresistive microfiber sensors. ACS Appl. Mater. Interfaces 2018, 10, 12773-12780. [CrossRef]

11. Cao, Z.; Wang, R.; He, T.; Xu, F.; Sun, J. Interface-controlled conductive fibers for wearable strain sensors and stretchable conducting wires. ACS Appl. Mater. Interfaces 2018, 10, 14087-14096. [CrossRef]

12. Zhang, Q.; Roach, D.J.; Geng, L.; Chen, H.; Qi, H.J.; Fang, D. Highly stretchable and conductive fibers enabled by liquid metal dip-coating. Smart Mater. Struct. 2018, 27, 035019. [CrossRef]

13. Luo, W.; Wu, T.; Chen, B.; Liang, M.; Zou, H. Highly stretchable conductors based on expanded graphite macroconfined in tubular rubber. ACS Appl. Mater. Interfaces 2017, 9, 43239-43249. [CrossRef]

14. Zhou, J.; Xu, X.; Xin, Y.; Lubineau, G. Coaxial Thermoplastic elastomer-wrapped carbon nanotube fibers for deformable and wearable strain sensors. Adv. Funct. Mater. 2018, 28, 1705591. [CrossRef]

15. Tang, Z.; Jia, S.; Shi, S.; Wang, F.; Li, B. Coaxial carbon nanotube/polymer fibers as wearable piezoresistive sensors. Sens. Actuators A Phys. 2018, 284, 85-95. [CrossRef]

16. Kwon, S.N.; Kim, S.W.; Kim, I.G.; Hong, Y.K.; Na, S.I. Direct 3D printing of graphene nanoplatelet/silver nanoparticle-based nanocomposites for multiaxial piezoresistive sensor applications. Adv. Mater. Technol. 2018, 3, 1800500. [CrossRef]

17. Wang, Y.; Chen, C.; Xie, H.; Gao, T.; Yao, Y.; Pastel, G.; Han, X.; Li, Y.; Zhao, J.; Fu, K.; et al. 3D-printed all-fiber Li-ion battery toward wearable energy storage. Adv. Funct. Mater. 2017, 27, 1703140. [CrossRef]

18. Kotikian, A.; Truby, R.L.; Boley, J.W.; White, T.J.; Lewis, J.A. 3D printing of liquid crystal elastomeric actuators with spatially programed nematic order. Adv. Mater. 2018, 30, 1706164. [CrossRef]

19. Wang, X.; Li, J.; Song, H.; Huang, H.; Gou, J. Highly stretchable and wearable strain sensor based on printable carbon nanotube layers/polydimethylsiloxane composites with adjustable sensitivity. ACS Appl. Mater. Interfaces 2018, 10, 7371-7380. [CrossRef]

20. Kim, S.; Oh, J.; Jeong, D.; Park, W.; Bae, J. Consistent and reproducible direct ink writing of eutectic gallium-indium for high-quality soft sensors. Soft Robot. 2018, 5, 601-612. [CrossRef]

21. Cheng, M.; Jiang, Y.; Yao, W.; Yuan, Y.; Deivanayagam, R.; Foroozan, T.; Huang, Z.; Song, B.; Rojaee, R.; Shokuhfar, T.; et al. Elevated-temperature 3D printing of hybrid solid-state electrolyte for Li-ion batteries. Adv. Mater. 2018, 30, 1800615. [CrossRef] 
22. Yue, Y.; Zhang, C.; Zhang, H.; Zhang, D.; Chen, X.; Chen, Y.; Zhang, Z. Rheological behaviors of fumed silica filled polydimethylsiloxane suspensions. Compos. Part A Appl. Sci. 2013, 53, 152-159. [CrossRef]

23. Bounos, G.; Andrikopoulos, K.S.; Karachalios, T.K.; Voyiatzis, G.A. Evaluation of multi-walled carbon nanotube concentrations in polymer nanocomposites by Raman spectroscopy. Carbon 2014, 76, 301-309. [CrossRef]

24. Christ, J.F.; Aliheidari, N.; Ameli, A.; Pötschke, P. 3D printed highly elastic strain sensors of multiwalled carbon nanotube/thermoplastic polyurethane nanocomposites. Mater. Des. 2017, 131, 394-401. [CrossRef]

25. Zhang, R.; Deng, H.; Valenca, R.; Jin, J.; Fu, Q.; Bilotti, E.; Peijs, T. Strain sensing behaviour of elastomeric composite films containing carbon nanotubes under cyclic loading. Compos. Sci. Technol. 2013, 74, 1-5. [CrossRef]

26. Muth, J.T.; Vogt, D.M.; Truby, R.L.; Mengüç, Y.; Kolesky, D.B.; Wood, R.J.; Lewis, J.A. Embedded 3D printing of strain sensors within highly stretchable elastomers. Adv. Mater. 2014, 26, 6307-6312. [CrossRef]

(C) 2019 by the authors. Licensee MDPI, Basel, Switzerland. This article is an open access article distributed under the terms and conditions of the Creative Commons Attribution (CC BY) license (http://creativecommons.org/licenses/by/4.0/). 